\title{
Successful pregnancy and delivery achieved following intracytoplasmic sperm injection using teratozoospermic sperm exhibiting marked dysplasia of the fibrous sheath: a case report
}

\author{
Masato Saito ${ }^{1}$, Takayuki Tatsumi ${ }^{1,2,3}$, Takashi Nakasuji ${ }^{4}$, Megumi Ibayashi $^{1}$, Tatsuya Harada ${ }^{4,5}$, \\ Tohru Mutoh $^{6}$, Chizuru Ito ${ }^{6}$, Akira Komiya ${ }^{7}$, Kenichiro Hiraoka ${ }^{1,2,4}$, Kiyotaka Kawai ${ }^{1,2,4}$ \\ ${ }^{1}$ Department of Reproductive Medicine, Kameda IVF Clinic Makuhari, Chiba, Japan; ${ }^{2}$ Department of Reproductive Medicine, Kameda Medical \\ Center, Chiba, Japan; ${ }^{3}$ Department of Pediatrics, Perinatal and Maternal Medicine (Ibaraki), Graduate School, Tokyo Medical and Dental University, \\ Tokyo, Japan; ${ }^{4}$ Comprehensive Reproductive Medicine, Regulation of Internal Environment and Reproduction, Graduate School, Tokyo Medical and \\ Dental University, Tokyo, Japan; ${ }^{5}$ Department of Reproductive Medicine, Kubonoya IVF Clinic, Chiba, Japan; ${ }^{6}$ Department of Functional Anatomy, \\ Reproductive Biology and Medicine, Graduate School of Medicine, Chiba University, Chiba, Japan; ${ }^{7}$ Department of Urology, Chiba University, \\ Graduate School of Medicine, Chiba, Japan \\ Correspondence to: Takayuki Tatsumi. 113 - 8510, 1-5-45 Yushima, Bunkyo-ku, Tokyo, Japan. Email: ttatcrm@tmd.ac.jp.
}

\begin{abstract}
A husband and his wife, both 34 years old, consulted our clinic because of primary infertility. Sperm analysis revealed that the sperm concentration, motility, and progressive motility were $(42.8 \pm 22.8) \times 10^{6} / \mathrm{mL}$, $23.3 \% \pm 12.2 \%$, and $12.9 \% \pm 6.1 \%$, respectively. Based on Krugar strict morphology criteria, $100 \%$ of the sperm were teratozoospermic, with 7.9\% DNA fragmentation index. Observation of the sperm under a transmission electron microscope revealed that most parts of the fibrous sheath (FS) surrounding the tails of the sperm were missing from midway through the principal piece to the end piece, although the sperm's heads, necks and midpieces were morphologically normal. To collect oocytes, the gonadotropin-releasing hormone antagonist protocol was carried out, and 7 oocytes were retrieved. Intracytoplasmic sperm injection (ICSI) was performed for all the teratozoospermic sperm. Of the 7 oocytes, 3 were fertilized, and one 8-cell embryo and 2 expanded blastocysts were vitrified. Although repeated transfers of expanded blastocysts resulted in no implantation, one 8-cell embryo transfer in a hormone replacement therapy cycle led to pregnancy. The pregnancy using an 8 -cell vitrified embryo resulted in the delivery of a healthy female baby at 38 weeks of gestation. No congenital malformations were found until 28 days after birth. Our results demonstrated that healthy birth could be achieved following the transfer of an embryo derived from ICSI using teratozoospermic sperm exhibiting the dysplasia of the fibrous sheath (DFS). Furthermore, while the previous reports on DFS have not investigated male infertility, we evaluated sperms from various aspects such as Kruger sperm function test, chromatin dispersion test, electron microscopy findings, time-lapse images of the obtained embryos, and concluded that ICSI could be desirable as a treatment policy for DFS.
\end{abstract}

Keywords: Fibrous sheath (FS); dysplasia of the fibrous sheath (DFS); intracytoplasmic sperm injection (ICSI); case report

Submitted Oct 24, 2019. Accepted for publication Dec 09, 2019.

doi: $10.21037 /$ tau.2019.12.14

View this article at: http://dx.doi.org/10.21037/tau.2019.12.14 


\section{Introduction}

Teratozoospermia is the morphological abnormality involving deformities of the head and tail of the sperm. Progress has been made in research investigating the association of teratozoospermia with sperm chromosomal abnormalities (1), however, there are only few reports on tail deformities contributing to teratozoospermia. Tail deformities can cause abnormal movement of the flagella, which may result in asthenozoospermia, leading to male infertility (2). Defects in the tail's fibrous sheath (FS) - the primary structure of the tail that encloses the axial filament of the sperm tail-contributes to sperm tail deformity (3). FS is required for sperm plasticity and is known to contribute to the generation of kinetic energy in sperm. As a result, defects in FS structure can cause abnormal sperm motility (2).

Patients with dysplasia of the fibrous sheath (DFS) exhibit reduced sperm motility, making fertilization difficult with conventional in vitro fertilization (IVF). When DFS is diagnosed, it may be best to consider properly formed sperm for use in intracytoplasmic sperm injection (ICSI) (4). In the presence of many poorly formed sperm, ICSI is generally performed with sperm of good morphology, but cases with $100 \%$ tail abnormalities are generally not anticipated. There are very few reports of successful deliveries using morphologically abnormal sperm in ICSI, and the reports are old, containing little information about the sperm. Here, we report a case exhibits primary infertility, in which $100 \%$ of the sperm exhibited DFS with marked tail thinning that started around their central region. Nevertheless, a successful pregnancy and delivery were achieved using these sperm following ICSI. We present the following case in accordance with the CARE Guideline (5).

\section{Case presentation}

A Japanese husband and his wife, both 34 years old, came to our clinic for conception. They had primary infertility for 1 year and 2 months by the time they were examined at our clinic. There was nothing notable in their family histories, and the husband's older brother and his female partner had a live birth from a natural pregnancy. The couple was performed timing intercourse once and IntraUterine Insemination twice but were unsuccessful. When the husband's sperm were examined under a microscope during a semen analysis, all of the sperm exhibited thinning that started around the middle of the sperm tail (Figure 1). Sperm motility and progressive motility were $23.3 \% \pm 12.2 \%$
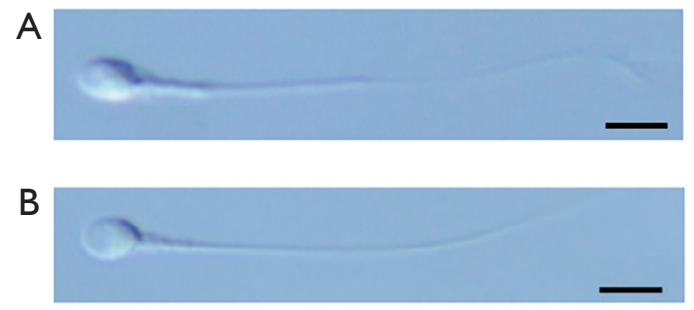

Figure 1 The patient's sperm and a sperm with normal morphology viewed with differential interference $(\times 600)$. (A) The patient's sperm starts to thin drastically starting around the middle of the tail, which continues to the end; (B) no areas of thinning can be seen in the sperm with normal morphology. Scale bars: $5 \mu \mathrm{m}$.

and $12.9 \% \pm 6.1 \%$ (mean \pm standard deviation), respectively. Both the values were below the standard thresholds defined by the World Health Organization (WHO) and indicated asthenozoospermia (Table 1). Semen volume and sperm concentration were within the reference range. Volumes of both the left and right testes were examined to be same $(24 \mathrm{~mL})$ with no abnormal findings in a scrotal ultrasonography. Presence of a varicocele was not detected. A blood test revealed that the levels of luteinizing hormone ( $4.2 \mathrm{mIU} / \mathrm{mL})$, follicle-stimulating hormone $(5.7 \mathrm{mIU} / \mathrm{mL})$, and total testosterone $(6.24 \mathrm{ng} / \mathrm{mL})$ were within the reference ranges. As pregnancy had not occurred with nonassisted reproductive technology (ART) treatments and the patient exhibited asthenozoospermia with morphological abnormalities of the sperm tails, we decided to perform ART. Furthermore, due to the sperm tail abnormalities and reduced motility rate, we considered fertilization with conventional IVF to be difficult and decided to use ICSI following previous studies $(6,7)$. We explained the treatment policy to the couple, mentioning the possibility that the abnormalities of the tail might be transmitted to the next generation, and informed consent was obtained.

\section{Methods}

\section{Semen analyses}

After 2-3 days of abstinence, the patient masturbated to collect 7 semen samples. After confirming liquefaction, semen volume, sperm concentration, motility rate, and progressive motility rate were measured based on the WHO's standards for semen analyses (8). To determine the proportion of deformed sperm, the man's sperm were smeared on a glass slide, and the dried specimens were 
Table 1 Test results of the 7 semen samples

\begin{tabular}{lcccccccc}
\hline Semen tests & 1 & 2 & 3 & 4 & 5 & 6 & 7 & Mean \pm SD \\
\hline Semen volume $(\mathrm{mL})$ & 2.0 & 2.4 & 2.0 & 1.2 & 3.6 & 2.2 & 4.5 & $2.6 \pm 1.0$ \\
Sperm concentration $\left(\times 10^{6} / \mathrm{mL}\right)$ & 26.9 & 54.5 & 83.0 & 16.4 & 62.5 & 20.6 & 35.5 & $42.8 \pm 22.8$ \\
Sperm motility $(\%)$ & 16.4 & 33.0 & 48.2 & 14.6 & 23.2 & 9.7 & 18.3 & $23.3 \pm 12.2$ \\
Sperm progressive motility $(\%)$ & 13.4 & 17.4 & 24.1 & 9.8 & 12.8 & 2.9 & 9.9 & $12.9 \pm 6.2$ \\
\hline
\end{tabular}

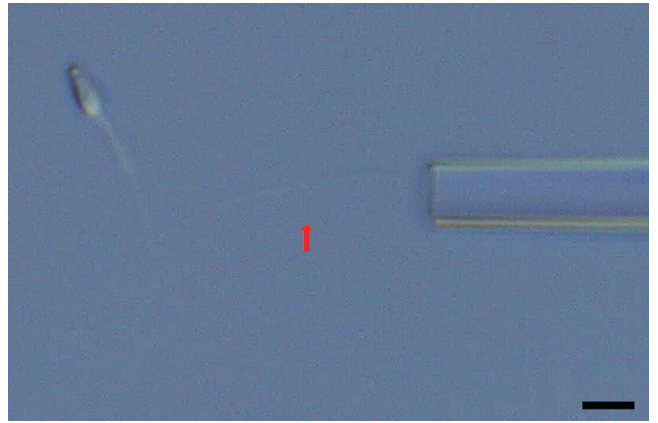

Figure 2 Sperm with a severed deformed tail. The arrow shows the sperm tail severed by a Piezo pulse. Scale bars: $5 \mu \mathrm{m}$.

stained using Diff-Quik ${ }^{\circledR}$ (Sysmex Corporation, Kobe, Japan). Two hundred of the stained sperm were counted following the WHO's Krugar strict criteria.

\section{Sperm chromatin dispersion test}

Three samples of semen-the patient's sperm and two control samples of sperm without any sperm deformitieswere examined by sperm chromatin dispersion test as described method with little modifications (9) (Supplementary 1).

\section{Transmission electron microscopy}

The patient's sperm and a control sample of the sperm with no clear deformities in a semen test were analyzed under a transmission electron microscope as described method with little modifications (10) (Supplementary 2).

\section{ICSI and culture methods}

ICSI was performed according to previously described methods (11). Sperm selection and sperm immobilization were performed in $7 \%$ PVP. Sperm immobilization was performed based on a previous report (12). After immobilization with the 2 Piezo pulses, the sperm with normal tail morphology generally appear bent but are not severed. However, in the present case, because of the presence of the thin regions in the sperm tails, the tails were more fragile than those with normal morphology and were easily severed by the Piezo pulses (Figure 2). When Piezo pulses were applied to the regions on the sperm tails that appeared to have normal thickness, the tails were not severed. Therefore, to avoid using sperm with severed tails in ICSI, Piezo pulses were only applied to the sperm with tails that appeared to have normal thickness, immobilizing them without severing their tails. Oocytes that underwent ICSI were cultured individually using a $\mu$ Drop GPS ${ }^{\circledR}$ (LifeGlobal, China) culture dish, $50 \mu \mathrm{L}$ of SAGE 1-Step (ORIGIO) culture medium, and a CCM-iBIS (Astec Co., Japan) incubator at $37{ }^{\circ} \mathrm{C}$, in an environment of $5 \% \mathrm{CO}_{2}$, $5 \% \mathrm{O}_{2}$, and $90 \% \mathrm{~N}_{2}$. Time lapse photographs were taken every 15 minutes after ICSI. Considering the day of the oocyte collection as day 0 , survival and fertilization were assessed on day 1 and then the oocytes cultured until day 5 and cryopreserved. Cryopreservation of embryos were performed according to previously described methods. A single vitrified-warmed embryo transfer was performed during a hormone replacement treatment cycle.

\section{Analysis of fertilized embryos and division speed}

Time lapse images of the 3 embryos that were fertilized with ICSI were analyzed retrospectively. With ICSI as the starting point, the time (hours) taken for a 2-cell, 4-cell, 5 -cell, morula, and a blastocyst formation were defined as T2, T4, T5, TM, and TB, respectively.

\section{Results}

\section{Semen analyses}

Table 1 shows the results of the 7 semen sample analyses. The motility rate was over $40 \%$ only in the case of the third 

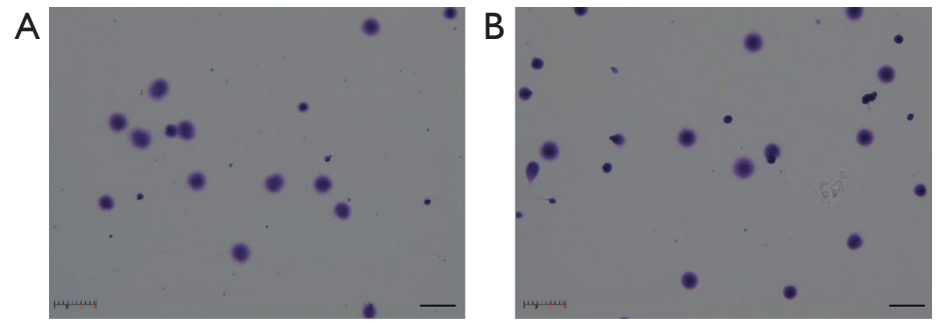

Figure 3 Sperm chromatin dispersion test. (A) Sperm chromatin dispersion test of the patient's sperm; (B) sperm chromatin dispersion test of the sperm with normal morphology. Scale bars: $20 \mu \mathrm{m}$.

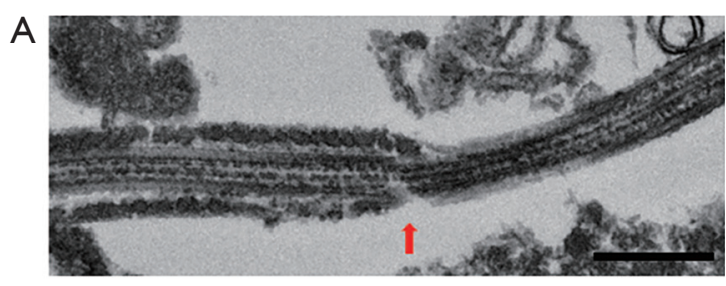

B

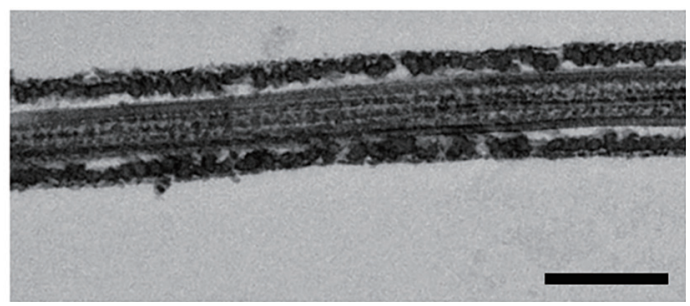

Figure 4 Electron microscope images of fibrous sheath defects in a sperm tail. (A) The fibrous sheath is truncated and partially missing in the patient's sperm; (B) the fibrous sheath is contiguous with no defects in the sperm with normal morphology. Scale bars: $500 \mathrm{~nm}$.

semen sample, with the other 6 samples showing motility rates and progressive motility rates much lower than the WHO standards (8).

Based on the Krugar strict criteria for categorizing the sperm, in the current case, all the sperm had abnormal tails, with a normal morphology rate of $0 \%$.

\section{Analysis of sperm chromatin dispersion test results}

The DNA fragmentation rate of the patient's semen and that of the two control samples were $7.6 \%, 5.5 \%$, and $6.9 \%$, respectively (Figure 3). No clear differences were found between the DNA fragmentation rates of the patient's semen and that of the control samples', suggesting that the tail deformities are not necessarily accompanied by abnormalities in sperm DNA.

\section{Analysis of transmission electron microscopy findings}

Observation of the patient's sperm under a transmission electron microscope showed that most parts of the FS surrounding the tails of the sperm were missing from midway through the principal piece to the end piece (Figure 4). A light microscope observation also showed thinning of the tails starting from the FS defects. Nothing abnormal was observed from the sperm head to the central fragment. The axial filaments of the sperm tails maintained their $9+2$ structure. Apart from the FS defects, no other structural abnormalities were observed (Figure 5).

\section{Analysis of the fertilized embryos and division speed}

Table 2 shows the time points for the normally fertilized embryos. Compared to the previous reports, no clear differences in the timings of embryonic development during pregnancy were observed for T2 (21.4-34.8 h), T3 (27.847.8 h), T4 (33.1-57.2 h), T5 (37.5-67.0 h), TM (57.6$102.8 \mathrm{~h})$, or TB (94.6-117.8 h) (13).

\section{Case course}

Ovarian stimulation was performed using the antagonist method for collecting oocytes. Nine oocytes were collected based on the transvaginal ultrasound. After preincubation, ICSI was performed on the 7 mature oocytes following 6 hours of collection. Considering the day of the oocyte collection as day 0 , fertilization was confirmed after 24-25 hours (day 1), and 3 fertilized oocytes that exhibited 2 pronucleus $(\mathrm{PN})$ were cultured until day 5. An 8-cell embryo (Veeck classification Grade 1) was vitrified for storage on day 3, and two blastocysts were vitrified on day 4 (Gardner classification 4BB) and day 5 (Gardner 4BC). Vitrified-warmed embryo transfer was performed during a hormone replacement treatment cycle. On day 5 

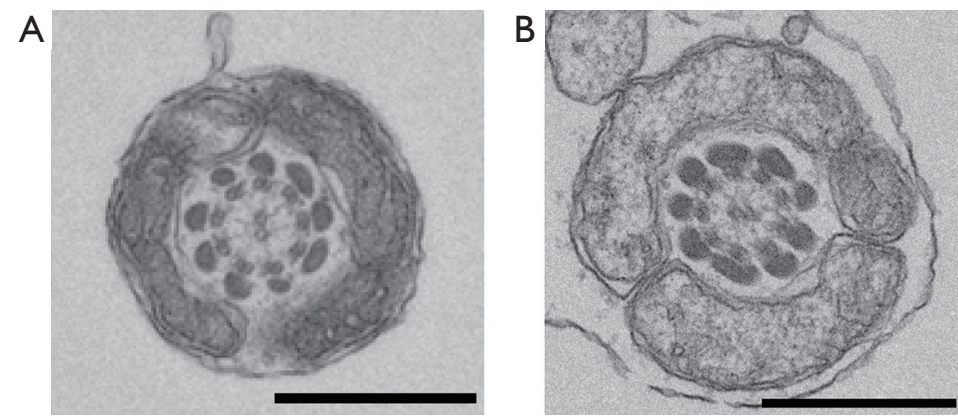

Figure 5 Short-axis electron microscope images of the patient's sperm and sperm with normal morphology. (A) The 9+2 structure of the axial filament is maintained in the patient's sperm; (B) a short-axis slice of sperm with normal morphology. Scale bars: $500 \mathrm{~nm}$.

Table 2 Time points (h) from intracytoplasmic sperm injection (ICSI) using vitrified-warmed embryos

\begin{tabular}{lcccccc}
\hline Embryo & T2 & T3 & T4 & T5 & TM & TB \\
\hline Grade 18-cell & 30.1 & 40.1 & 40.4 & 50.6 & - & 79.9 \\
Grade 4BB & 22.9 & 31.9 & 32.4 & 43.1 & 86.6 \\
Grade 4BC & 25.7 & 35.7 & 36.9 & 48.7 & 86.2 & 96.7 \\
\hline
\end{tabular}

after starting luteal support, both the blastocysts were transferred, but pregnancy could not be achieved. On day 3 after starting luteal support, an 8-cell embryo was transferred, and on day 12 after the transfer, a positive pregnancy response (serum human chorionic gonadotropin $242.6 \mathrm{mIU} / \mathrm{mL}$ ) was obtained, and two weeks later, 1 fetal heartbeat was confirmed in the uterus. The pregnancy was normal, and the patient gave birth to a healthy $3,400 \mathrm{~g}$ female baby at 38 weeks' gestation. Till 1 month, the neonate exhibited no clear abnormalities (14).

\section{Discussion}

DFS in the present case did not affect the fragmentation rate of the sperm DNA. Furthermore, despite $100 \%$ of the sperm being deformed and exhibiting a reduced motility rate, no abnormality in the testicular volume or hormone levels could be found, and successful pregnancy was achieved through ICSI. This case demonstrates that ICSI can be an effective method of fertilization in cases of sperm with DFS.

The FS of sperm tails in mammals is a cytoskeletal component that surrounds the axial filament with a $9+2$ arrangement of microtubules and 9 outer dense fibers and is known to play a variety of roles (15). The number of functions of the FS that have so far been discovered (2) are as follows: (I) the FS protects the axial filament and controls the sperm's flagellar movement, (II) it transmits calcium signals via proteins such as A-kinase-anchoring protein (AKAP) 4 and AKAP3, (III) a glycolysis enzyme that exists in the FS-glyceraldehyde 3-phosphate dehydrogenase (GAPDH)-takes part in hyperactivation of the sperm. Owing to the above functions, FS defects are expected to cause a variety of symptoms. In a study of AKAP4 knockout mice, the outer dense fibers of the sperm were preserved in the absence of FS, which resulted in abnormal flagellar movements causing infertility (16).

DFS in human sperm was first reported by Chemes et al. in 1987 as a hereditary factor (3). Mutations of genes involved in the FS formation can cause thinning or hyperplasia of the sperm principal piece, causing the tail to shorten or thicken (4). The present case did not exhibit any clear familial factors and was considered as an isolated occurrence. Moreover, conventional IVF has been reported to be unsuccessful in producing fertilized oocytes using sperm with DFS, indicating that this may not be an effective technique in such cases $(4,6,7,17)$. Based on these reports, we chose ICSI, which resulted in lower $2 \mathrm{PN}$ rate while having no effects on the normal development of the fertilized oocytes. In addition, transfer of an early embryo resulting in a successful pregnancy and delivery of a healthy baby indicates that ICSI can be an effective technique in 
patients with DFS.

Progress has been made in research involving the sperm deformity and its association with the rates of DNA fragmentation. The DNA fragmentation rate in the present case was $7.6 \%$, which is not much different than that of the control specimens measured at the same time $5.5 \%$ and $6.9 \%$ ) and is much lower than the level above which natural pregnancy is thought to be difficult (30\%) (18). This suggests that, DFS may not affect DNA fragmentation. Furthermore, no obvious abnormalities in the testicular volume or hormone levels were observed in this case, and a fertilized embryo developed through pregnancy, leading to delivery with no abnormalities. Apart from the reduced motility rate accompanying the tail deformity, there were no other notable findings. While the previous reports on DFS did not investigate male infertility, our case may serve as a helpful reference going forward.

To date, there are 4 reports on patients with DFS whose sperm were used in successful deliveries $(6,7,17,19)$. These results show that a wide variety of morphologies of sperm with DFS are observed. Usually, the sperm with nearly normal morphology can be selected and used in ICSI. In this case and in other births from sperm with DFS, all the babies are reported to be healthy with no chromosomal abnormalities or deformities. This indicates that the sperm with DFS are still fertile and can result in healthy deliveries, without causing major deformities in the child. In addition, it cannot be said that this $100 \%$ of the sperm exhibited DFS could not be fertilized with conventional IVF, but ICSI is considered desirable as a treatment policy.

In the present case, 2 blastocysts could not be implanted, but an 8-cell embryo was transferred. Generally, spermderived activation occurs in the latter half of embryonic development (20). We retrospectively examined the time points in embryonic development to determine whether the use of the deformed sperm had any negative effects on growth. The 2 blastocysts that could not be implanted and the one 8-cell embryo that was implanted were from $\mathrm{T} 2$ to $\mathrm{TB}$ and did not exhibit any clear differences in the timings of their development compared to what are considered as healthy embryos. We were concerned that the use of deformed sperm in the present case might lead to abnormalities in the latter stages of embryonic development, but the blastocysts developed without any difficulties.

In the present case, other than reduced motility rates due to tail deformities, no other abnormal findings were observed in the sperm with DFS, and ICSI resulted in the birth of a healthy female baby. Based on the findings of our study, we postulate that while developing a treatment plan with cases of infertility related to DFS, waste of time on attempting a natural pregnancy or insemination may be avoided by selecting ICSI as the first option.

\section{Acknowledgments}

Funding: None.

\section{Footnote}

Conflicts of Interest: All authors have completed the ICMJE uniform disclosure form (available at http://dx.doi. org/10.21037/tau.2019.12.14). The authors have no conflicts of interest to declare.

Ethical Statement: The authors are accountable for all aspects of the work in ensuring that questions related to the accuracy or integrity of any part of the work are appropriately investigated and resolved. The protocols for the research project including human subjects have been approved by Tokyo Medical Dental University (approval number G 2018 - 033). Informed consent was obtained directly from the patient.

Open Access Statement: This is an Open Access article distributed in accordance with the Creative Commons Attribution-NonCommercial-NoDerivs 4.0 International License (CC BY-NC-ND 4.0), which permits the noncommercial replication and distribution of the article with the strict proviso that no changes or edits are made and the original work is properly cited (including links to both the formal publication through the relevant DOI and the license). See: https://creativecommons.org/licenses/by-nc$\mathrm{nd} / 4.0 /$.

\section{References}

1. Gandini L, Lombardo F, Paoli D, et al. Study of apoptotic DNA fragmentation in human spermatozoa. Hum Reprod 2000;15:830-9.

2. Lehti MS, Sironen A. Formation and function of sperm tail structures in association with sperm motility defects. Biol Reprod 2017;97:522-36.

3. Chemes HE, Carizza C, Scarinci F, et al. Lack of a head in human spermatozoa from sterile patients: a syndrome associated with impaired fertilization. Fertility and Sterility 1987;47:310-6. 
4. Chemes HE, Olmedo SB, Carrere C, et al. Ultrastructural pathology of the sperm flagellum: association between flagellar pathology and fertility prognosis in severely asthenozoospermic men. Hum Reprod 1998;13:2521-6.

5. Riley DS, Barber MS, Kienle GS, et al. CARE guidelines for case reports: explanation and elaboration document. J Clin Epidemiol 2017;89:218-35.

6. Stalf T, Sanchez R, Kohn FM, et al. Pregnancy and birth after intracytoplasmic sperm injection with spermatozoa from a patient with tail stump syndrome. Hum Reprod 1995;10:2112-4.

7. Olmedo SB, Rawe VY, Nodar FN, et al. Pregnancies established through intracytoplasmic sperm injection (ICSI) using spermatozoa with dysplasia of fibrous sheath. Asian J Androl 2000;2:125-30.

8. World Health Organization. Laboratory Manual for the Examination and Processing of human semen. 5th. Geneva, Switzerland ed. World Health Organization; 2010.

9. Fernandez JL, Muriel L, Rivero MT, et al. The sperm chromatin dispersion test: a simple method for the determination of sperm DNA fragmentation. J Androl 2003;24:59-66.

10. Tatsumi T, Takayama K, Ishii S, et al. Forced lipophagy reveals that lipid droplets are required for early embryonic development in mouse. Development 2018. doi: 10.1242/ dev.161893.

11. Hiraoka K, Kitamura S. Clinical efficiency of Piezo-ICSI using micropipettes with a wall thickness of $0.625 \mu \mathrm{m}$. J Assist Reprod Genet 2015;32:1827-33.

12. Hiraoka K, Otsuka Y, Kawai K, et al. Effects of sperm

Cite this article as: Saito $M$, Tatsumi T, Nakasuji T, Ibayashi M, Harada T, Mutoh T, Ito C, Komiya A, Hiraoka K, Kawai K. Successful pregnancy and delivery achieved following intracytoplasmic sperm injection using teratozoospermic sperm exhibiting marked dysplasia of the fibrous sheath: a case report. Transl Androl Urol 2020;9(2):800-806. doi: 10.21037/ tau.2019.12.14 direction (head first injection, tail first injection) in Piezo-ICSI on oocyte survival, fertilization and embryo morphokinetics in humans. Obstetrical and Gynecological Practice 2019;68:1145-53.

13. Chamayou S, Patrizio P, Storaci G, et al. The use of morphokinetic parameters to select all embryos with full capacity to implant. J Assist Reprod Genet 2013;30:703-10.

14. Hirahara F. Monitoring of of birth defects in Japan. Grants-in-Aid for Sci Res Jpn 2013.

15. Toshimori K, Ito C, Maekawa M, et al. Impairment of spermatogenesis leading to infertility. Anat Sci Int 2004;79:101-11.

16. Miki K, Willis WD, Brown PR, et al. Targeted disruption of the Akap4 gene causes defects in sperm flagellum and motility. Dev Biol 2002;248:331-42.

17. Mitchell V, Rives N, Albert M, et al. Outcome of ICSI with ejaculated spermatozoa in a series of men with distinct ultrastructural flagellar abnormalities. Hum Reprod 2006;21:2065-74.

18. Evenson DP, Larson KL, Jost LK. Sperm chromatin structure assay: its clinical use for detecting sperm DNA fragmentation in male infertility and comparisons with other techniques. J Androl 2002;23:25-43.

19. Olmedo SB, Nodar F, Chillik C, et al. Successful intracytoplasmic sperm injection with spermatozoa from a patient with dysplasia of the fibrous sheath and chronic respiratory disease. Hum Reprod 1997;12:1497-9.

20. Zheng WW, Song G, Wang QL, et al. Sperm DNA damage has a negative effect on early embryonic development following in vitro fertilization. Asian J Androl 2018;20:75-9. 


\section{Supplementary 1}

\section{Sperm chromatin dispersion test}

Three samples of semen-the patient's sperm and two control samples of sperm without any sperm deformities-were examined by sperm chromatin dispersion test. For each sample, $0.2 \mathrm{~mL}$ aliquots of raw semen and sperm isolated using the SpermGrad ${ }^{\mathrm{TM}}$ (Vitrolife Sweden AB, V. Frölunda, Sweden) density gradient in modified human tubal fluid medium were analyzed according to previously described method with little modifications (9). The aliquots were used directly or frozen in liquid nitrogen before analysis. After thawing at room temperature, the samples were diluted in phosphate buffered saline ( $\mathrm{pH}$ 7.4) medium to obtain sperm concentrations ranging between 20 and 30 million/ $\mathrm{mL}$. To obtain a $0.7 \%$ final agarose concentration, the sperm suspensions were mixed with $1 \%$ low-melting point aqueous agarose at $37^{\circ} \mathrm{C}$. Aliquots of $5 \mu \mathrm{L}$ from the mixture were pipetted onto a glass slide precoated with $0.65 \%$ standard agarose. It was dried at $80{ }^{\circ} \mathrm{C}$, covered with a coverslip $(18 \times 32 \mathrm{~mm})$, and left to solidify at $4{ }^{\circ} \mathrm{C}$ for 4 minutes. Similar to the halo test or comet assay, this agarose matrix allows to experiment with unfixed sperm on a slide in a suspension-like environment. After careful removal of the coverslips, the slides were immediately immersed horizontally in a tray with freshly prepared acid denaturation solution $(0.08 \mathrm{~N} \mathrm{HCl})$ for 7 minutes at $22{ }^{\circ} \mathrm{C}$ in the dark to generate restricted single-stranded DNA (ssDNA) motifs from DNA breaks. The denaturation was then stopped, and proteins were removed by a transfer of the slides to a tray with neutralizing and lysing solution 1 (0.4 M Tris, $0.8 \mathrm{M}$ DTT, 1\% SDS, and $50 \mathrm{mM}$ EDTA, $\mathrm{pH}$ 7.5) for 10 minutes at room temperature, which was followed by incubation in neutralizing and lysing solution $2(0.4 \mathrm{M}$ Tris, $2 \mathrm{M} \mathrm{NaCl}$, and $1 \% \mathrm{SDS}$, pH 7.5) for 5 minutes at room temperature. Slides were thoroughly washed in Trisborate-EDTA buffer (0.09 M Tris-borate and 0.002 M EDTA, pH 7.5) for 2 minutes, dehydrated in sequential 70\%, 90\%, and 100\% ethanol baths ( 2 minutes each), and air dried. Cells were stained with Wright staining solution (Merck UN1230, Darmstadt, Germany). After washing the slides in running water, the samples were embedded with Eukitt (O-Kinder, Breisgau, Germany) for brightfield microscopy. 


\section{Supplementary 2}

\section{Transmission electron microscopy}

The patient's sperm and a control sample of the sperm with no clear deformities in a semen test were analyzed under a transmission electron microscope. The sandwiched samples with the copper disks were frozen in liquid propane at $-175^{\circ} \mathrm{C}$, following which the samples were freeze substituted with $2 \%$ glutaraldehyde, $1 \%$ tannic acid in ethanol, and $2 \%$ distilled water at $-80{ }^{\circ} \mathrm{C}$ for 2 days. Afterwards, the samples were kept at $-20{ }^{\circ} \mathrm{C}$ for $3 \mathrm{~h}$, followed by warming up to $4{ }^{\circ} \mathrm{C}$ for $4 \mathrm{~h}$. Next, the samples were dehydrated by treating with ethanol 3 times, each wash lasting for $30 \mathrm{~min}$. It was followed by overnight dehydration with ethanol at room temperature. Following overnight dehydration, the samples were infiltrated with propylene oxide (PO) for 2 times (30 min each) and were put into a 70:30 mixture of PO and resin (Quetol-651, Nisshin EM Co., Tokyo, Japan) for $1 \mathrm{~h}$. The cap of the tube was kept open overnight to remove PO by volatilization. Next, the samples were transferred to a freshly made $100 \%$ resin and polymerized at $60{ }^{\circ} \mathrm{C}$ for $48 \mathrm{~h}$. The ultrathin $(70 \mathrm{~nm})$ sections were prepared with a diamond knife using an ultramicrotome (Ultracut UCT, Leica, Vienna, Austria). The sections were mounted on copper grids and stained with $2 \%$ uranyl acetate at room temperature for $15 \mathrm{~min}$. Next, the sections were washed with distilled water, followed by secondary staining with lead stain solution (Sigma-Aldrich Co., Tokyo, Japan) at room temperature for $3 \mathrm{~min}$. The grids were observed under a transmission electron microscope (JEM-1400Plus, JEOL Ltd., Tokyo, Japan) at an acceleration voltage of $100 \mathrm{kV}$. Digital images (3,296×2,472 pixels) were taken with a CCD camera (EM-14830RUBY2, JEOL Ltd., Tokyo, Japan). 